\title{
Biolumineszenz
}

\section{Autonome Lichtproduktion in Bakterien mit optimiertem ilux-Operon}

\section{CAROLA GREGOR}

ABTEILUNG NANOBIOPHOTONIK, MAX-PLANCK-INSTITUT FÜR BIOPHYSIKALISCHE CHEMIE, GÖTTINGEN

\section{Bioluminescence emission allows the direct visualization of living cells} without external light. Its generation usually requires addition of a luciferin, except for bacterial bioluminescence with the lux operon where synthesis and recycling of the luciferin are performed by enzymatic reactions in the cell. An improved ilux operon produces brighter bioluminescence and enables autonomous bioluminescence imaging with increased sensitivity, allowing the observation of single Escherichia coli cells.

DOI: $10.1007 / \mathrm{s} 12268-018-0976-8$

(c) Die Autorin

Die biochemische Erzeugung von Licht in lebenden Zellen wird als Biolumineszenz bezeichnet. Viele unterschiedliche Lebewesen können dadurch im Dunkeln leuchten. Neben Glühwürmchen zählen hierzu mehrere Arten von Quallen, Tiefseefischen, Pilzen, Dinoflagellaten und Bakterien. Das Biolumineszenzlicht wird durch die enzymatische Oxidation eines Luciferinmoleküls erzeugt. Dabei entsteht ein Produkt in einem elektronisch angeregten Zustand, das anschließend bei der Rückkehr in den Grundzustand ein Photon aussendet. Diese Reaktion katalysiert das Enzym Luciferase. Obwohl alle bekannten Biolumineszenzreaktionen nach diesem Prinzip ablaufen, sind die in der Natur vorkommenden Luciferine und die dazugehörigen Luciferasen strukturell sehr verschieden. Vermutlich hat sich also die Biolumineszenz im Laufe der Evolution auf mehreren Wegen unabhängig voneinander entwickelt [1]. Das Biolumineszenzlicht erfüllt je nach Organismus unterschiedliche Aufgaben und dient beispielsweise dem Anlocken von Beute oder zur Abschreckung [1].

Ähnlich wie Fluoreszenz kann Biolumineszenz zum Markieren und Abbilden lebender Zellen verwendet werden. Hierfür wird in der Regel die Luciferase in den Zellen hergestellt und das entsprechende Luciferin von außen zugegeben. Da das Luciferin durch die Biolumineszenzreaktion verbraucht wird, muss

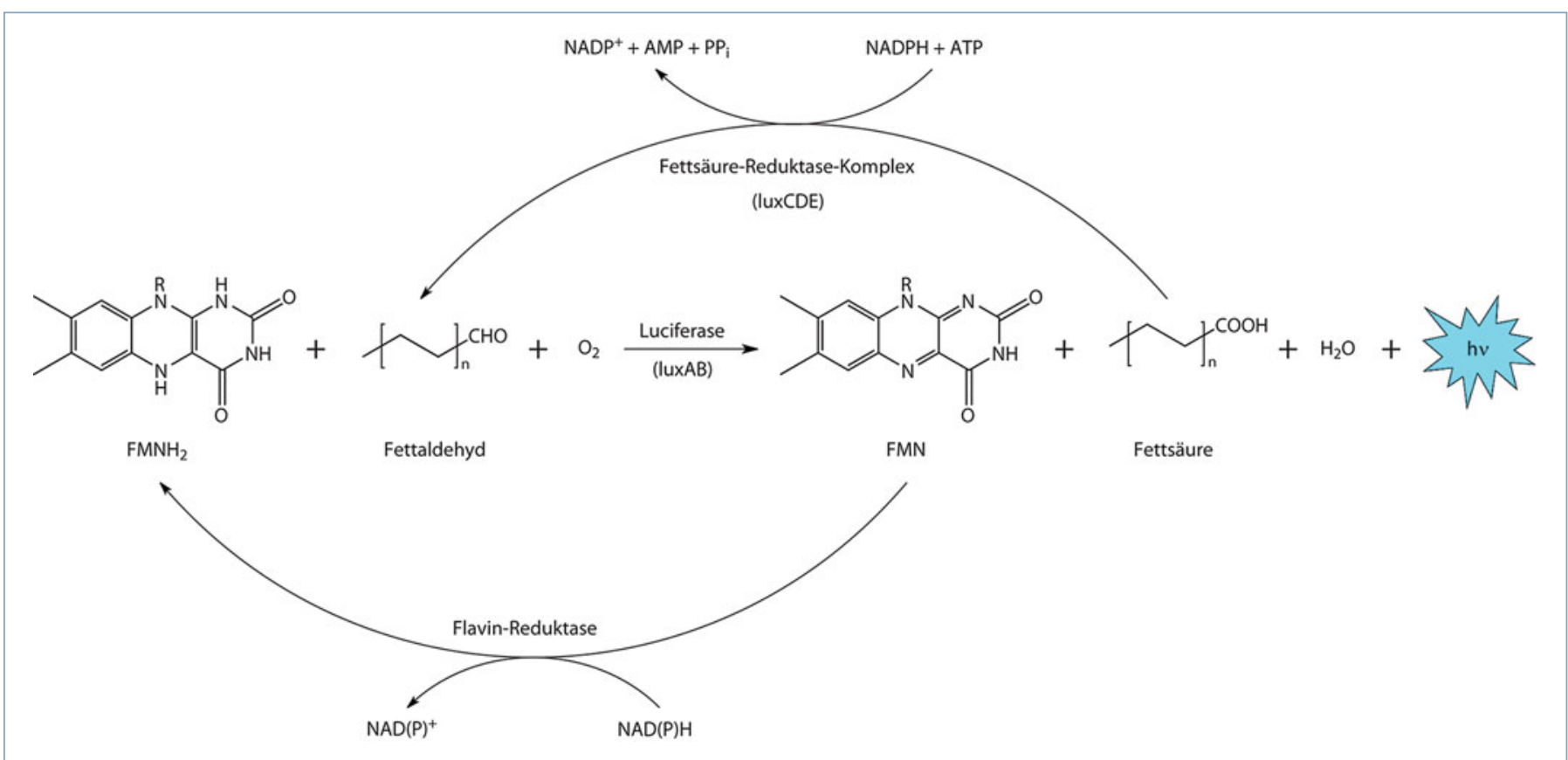

$\Delta$ Abb. 1: Bakterielle Biolumineszenzreaktion. Die Luciferase katalysiert die Umsetzung von FMNH ${ }_{2}$ und einem langkettigen Aldehyd ( $\mathrm{n} \geq 3$ [6]) mit molekularem Sauerstoff, wobei das Biolumineszenzlicht erzeugt wird. Die Substrate werden vom Fettsäure-Reduktase-Komplex und einer Flavin-Reduktase regeneriert. R: Ribitylseitenkette. 

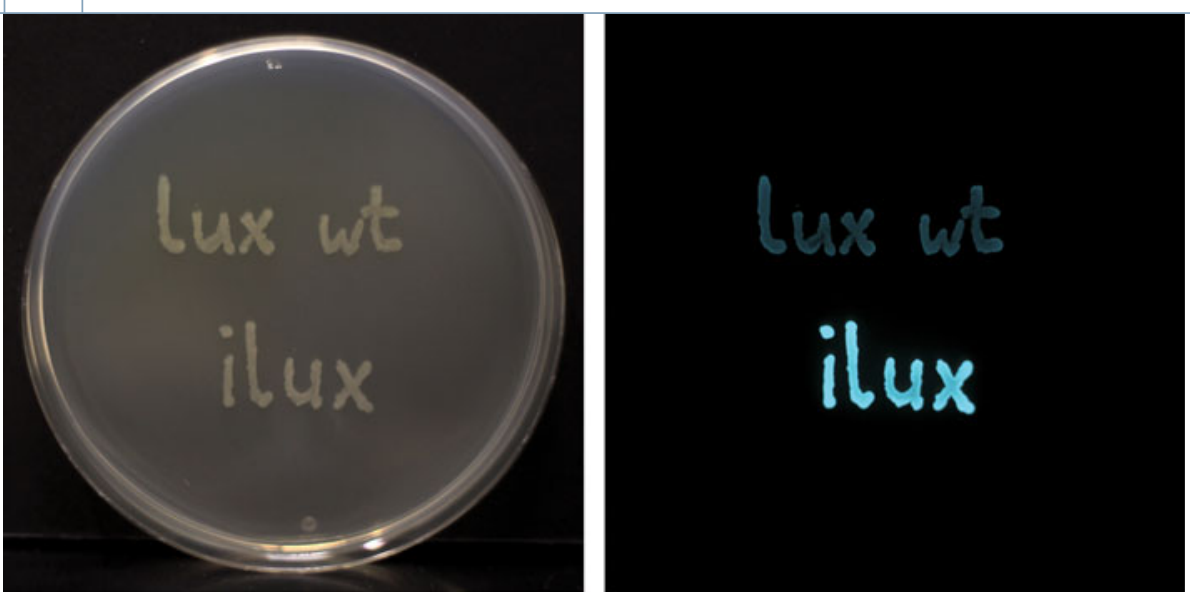

$\Delta$ Abb. 2: Escherichia coli-Bakterien mit dem ursprünglichen luxCDABE-Operon (oben) und dem verbesserten ilux-Operon (unten). Links: Aufnahme mit Raumlicht, rechts: abgegebenes Biolumineszenzlicht, im Dunkeln aufgenommen.

bei dieser Vorgehensweise eine relativ große Menge davon eingesetzt werden. Weitere Probleme sind unter anderem die nicht-enzymatische Oxidation des Luciferins in Lösung, wodurch sich das Hintergrundsignal erhöhen kann, und die zeitliche Veränderung des Biolumineszenzsignals bei Langzeitmessungen $[2,3]$. Ein anderer Ansatz ist die Herstellung und Rückgewinnung des Luciferins durch enzymatische Reaktionen in der Zelle, wie sie auch in der Natur stattfinden. Allerdings ist für die meisten Biolumineszenzsysteme bisher nicht vollständig bekannt, welche Proteine an diesen Reaktionen beteiligt sind. Eine Ausnahme stellt die Biolumineszenz in Bakterien dar. Hier werden sämtliche Proteine, die für die Aufrechterhaltung der Lichtproduktion erforderlich sind, in einem einzigen Operon, dem lux-Operon, codiert. Alle biolumineszierenden Bakterien verwenden als Luciferin reduziertes Flavinmononukleotid $\left(\mathrm{FMNH}_{2}\right)$, das in Form eines Zwischenprodukts während der Biolumineszenzreaktion ein Photon aussendet (Abb. 1). Gleichzeitig wird ein langkettiger Aldehyd, beispielsweise Tetradecanal, zur entsprechenden Säure oxidiert. Im lux-Operon sind neben den beiden Untereinheiten der Luciferase (codiert von den Genen $\operatorname{lux} A$ und $l u x B$ ) Enzyme für die Rückreduktion der Carbonsäure (luxCDE) und in den meisten Fällen auch für die Reduktion des oxidierten FMNs enthalten [4]. Da auch viele nicht-biolumineszierende Lebewesen wie Escherichia coli FMN- bzw. Flavin-Reduktasen produzieren, ist das Einbringen der luxCDABE-Gene zur Biolumineszenzerzeugung ausreichend.

\section{Helligkeitsverbesserung der bakteriellen Biolumineszenz}

Obwohl die bakterielle Biolumineszenz den Vorteil der Unabhängigkeit von äußeren Substraten besitzt, ist ihre Anwendbarkeit auf- grund der relativ geringen Helligkeit begrenzt. Daher ist eine Steigerung ihrer Helligkeit für die Verwendung als Reportersystem von besonderem Interesse. $\mathrm{Zu}$ diesem Zweck brachten wir das luxCDABE-Operon aus Photorhabdus luminescens, einem insektenpathogenen Bakterium, in E. coli-Zellen ein. Im nächsten Schritt erweiterten wir das luxCDABE-Operon um das frp-Gen aus dem biolumineszenten Bakterium Vibrio campbellii. Dieses Gen codiert eine Flavin-Reduktase und bewirkt eine Steigerung der $\mathrm{FMNH}_{2}$-Produktion in E. coli. Anschließend veränderten wir in mehreren Screening-Runden sämtliche Gene des neuen Operons abwechselnd durch Zufallsmutagenese und verwendeten die jeweils hellsten Varianten für den nächsten Mutageneseschritt. Das Ergebnis ist das optimierte Operon ilux [5], das verglichen mit dem ursprünglichen luxCDABE-Operon eine siebenmal höhere Biolumineszenz in E. coliZellen erzeugt (Abb. 2). Eine maximale Lichtausbeute wird in E. coli bei $37{ }^{\circ} \mathrm{C}$ erreicht, während die Helligkeit bei $22{ }^{\circ} \mathrm{C}$ um einen Faktor 2-3 geringer ist.

\section{Biolumineszenzmikroskopie einzelner Bakterien}

Die verbesserte Lichtausbeute mit dem iluxOperon kann unter anderem verwendet werden, um die Biolumineszenz einzelner Bakterien mikroskopisch aufzunehmen. Hierfür werden die resuspendierten Bakterien zwischen einem Deckgläschen und einem Agarstück mit Kulturmedium eingebettet, um eine Bewegung der Zellen zu verhindern. Für die Beobachtung der Bakterien wird ein Mikroskop mit einer lichtempfindlichen Kamera benötigt. Im Gegensatz zur Fluoreszenzmikroskopie ist für die Biolumineszenzdetektion kein Anregungslicht erforderlich, was ein Bleichen der Probe vermeidet. Daher können die Zellen über beliebig lange Zeit beob- achtet werden, solange die Zelldichte nicht zu hoch wird und eine ausreichende Nährstoff- und Sauerstoffversorgung gewährleistet ist. Abbildung 3A zeigt die Biolumineszenz einzelner ilux-exprimierender E. coliBakterien über eine Zeit von zehn Stunden. Deutlich zu erkennen sind das Wachstum und die Teilung der Zellen über mehrere Generationen, bis schließlich eine durchgehende Zellschicht auf dem Deckgläschen entsteht.

\section{Toxizitätsmessungen auf Einzelzellebene}

Da die Biolumineszenzreaktion indirekt zelluläre Energie in Form von ATP und NADPH benötigt, verschwindet das Signal beim Zelltod. Dies ermöglicht die Untersuchung des Einflusses von Toxinen auf die Lebensfähigkeit der Zellen. Aufgrund der erhöhten Helligkeit sind solche Messungen mit dem iluxOperon auch auf Einzelzellebene durchführbar, wodurch individuelle Unterschiede zwischen einzelnen Bakterien der Population direkt sichtbar sind. Als Beispiel hierfür ist in Abbildung 3B die Wirkung des Aminoglykosid-Antibiotikums Kanamycin gezeigt. Dieses Antibiotikum hemmt die Proteinsynthese und führt zunächst zum Einstellen der Zellteilung. Wie der Verlauf des Biolumineszenzsignals zeigt, bleiben die Zellen jedoch über längere Zeit metabolisch aktiv und sterben erst im Laufe mehrerer Tage, bis schließlich nach fünf Tagen das Signal fast vollständig verschwunden ist.

\section{Weitere Anwendungsgebiete}

Durch Biolumineszenz können lichtempfindliche Prozesse, wie z. B. circadiane Rhythmik, beobachtet werden, während dies mit Fluoreszenz aufgrund des intensiven Anregungslichtes nicht möglich ist. Darüber hinaus bietet die bakterielle Biolumineszenz neben den bereits erwähnten Toxizitätsmessungen verschiedene weitere Anwendungsmöglichkeiten. Hierzu zählen beispielsweise die Verwendung des lux-Operons als Reporter für Genexpressionsanalysen und in vivo-Bildgebung von Tumoren oder der Ausbreitung pathogener Bakterien im Wirtsorganismus. Die verbesserte Helligkeit des ilux-Operons ermöglicht dabei eine höhere Sensitivität und ist dadurch insbesondere für Einzelzellmessungen von Vorteil.

\section{Danksagung}

Mein besonderer Dank gilt Stefan W. Hell für die Ermöglichung dieser Arbeit in seiner Abteilung. 


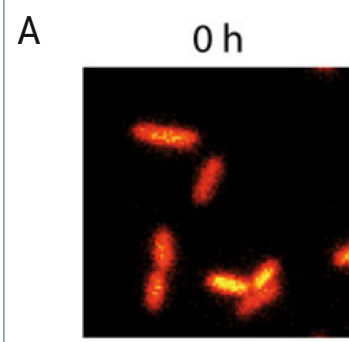

B

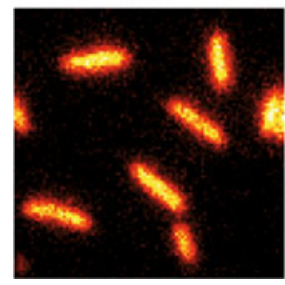

$2 \mathrm{~h}$

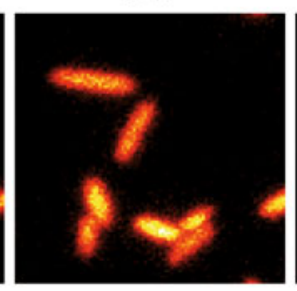

$6 \mathrm{~h}$

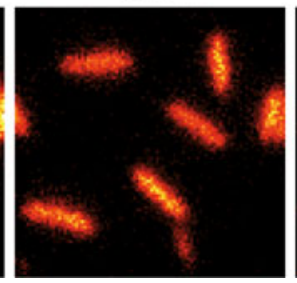

$4 \mathrm{~h}$

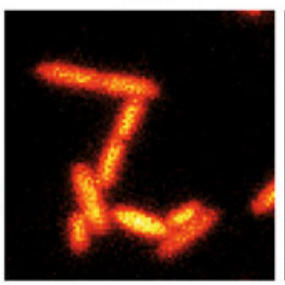

$12 \mathrm{~h}$

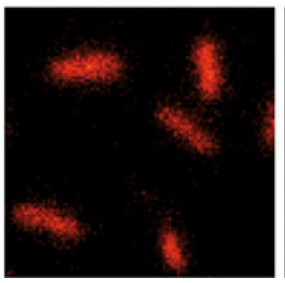

$6 \mathrm{~h}$

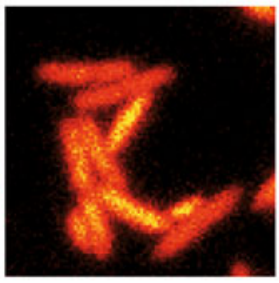

$24 \mathrm{~h}$

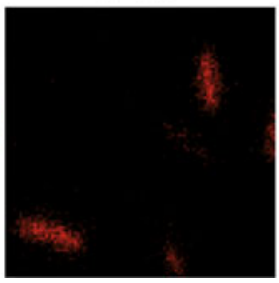

$8 \mathrm{~h}$

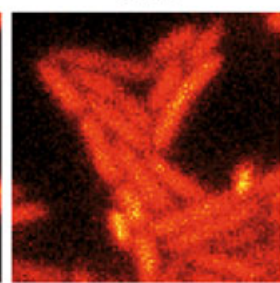

$48 \mathrm{~h}$
$10 \mathrm{~h}$

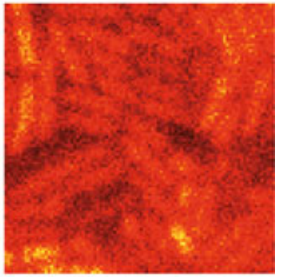

$120 \mathrm{~h}$

$\triangle$ Abb. 3: Biolumineszenz einzelner ilux-exprimierender Escherichia coli-Zellen, aufgenommen bei Raumtemperatur mit einer Aufnahmedauer von zehn Minuten pro Bild. A, Teilung der Zellen in Abwesenheit von Toxinen. B, abnehmendes Signal bei Zelltod, der durch Kanamycin (50 $\mu$ g/ml) induziert wurde. Die Farbskala zeigt die Helligkeit von minimalen (schwarz) bis zum maximalen Wert (weiß).

\section{Literatur}

[1] Haddock SH, Moline MA, Case JF (2010) Bioluminescence in the sea. Annu Rev Mar Sci 2:443-493

[2] Zhao H, Doyle TC, Wang RJ et al. (2004) Characterization of coelenterazine analogs for measurements of Renilla luciferase activity in live cells and living animals.

Mol Imaging 3:43-54

[3] Inoue Y, Sheng F, Kiryu S et al. (2011) Gaussia luciferase for bioluminescence tumor monitoring in comparison with firefly luciferase. Mol Imaging 10:377-385

[4] Dunlap P (2014) Biochemistry and Genetics of Bacterial Bioluminescence. In: Thouand G, Marks R (Hrsg)

Bioluminescence: Fundamentals and Applications in Biotechnology (Band 1). Springer, Berlin, S 37-64 [5] Gregor C, Gwosch KC, Sahl SJ et al. (2018) Strongly enhanced bacterial bioluminescence with the ilux operon for single-cell imaging. Proc Natl Acad Sci USA 115:962-967 [6] Hastings JW, Spudich J, Malnic G (1963) The influence of aldehyde chain length upon the relative quantum yield of the bioluminescent reaction of Achromobacter fischeri.

J Biol Chem 238:3100-3105
Open Access:

This article is distributed under the terms of the Creative Commons Attribution 4.0 International License (http://creativecommons.org/licenses/by/4.0/), which permits use, duplication, adaption, distribution and reproduction in any medium or format, as long as you give appropriate credit to the original author(s) and the source, provide a link to the Creative Commons license, an

Open access fundig provided by Max Planck Society.

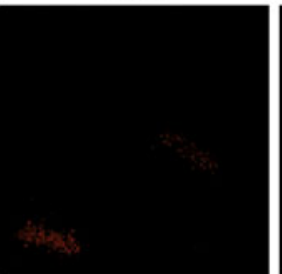

Dr. Carola Gregor

Am Fassberg 11

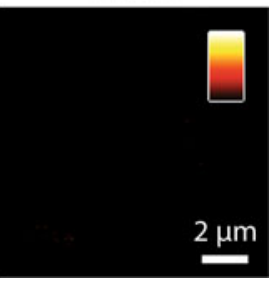

\section{Korrespondenzadresse:}

Max-Planck-Institut für biophysikalische Chemie

Abteilung NanoBiophotonik

D-37077 Göttingen

Tel.: 0551-201-2567

Fax: 0551-201-2505

carola.gregor@mpibpc.mpg.de

www.mpibpc.mpg.de/de/hell

Carola Gregor
2006-2011 Biochemiestudium an der Universität und Medizinischen Hochschule
Hannover. 2011-2016 Promotion am Max-Planck-Institut für biophysikalische Chemie,
Göttingen (Doktorgrad verliehen von der Universiät Hannover). Seit 2016 Postdoc am
Max-Planck-Institut für biophysikalische Chemie, Göttingen.

